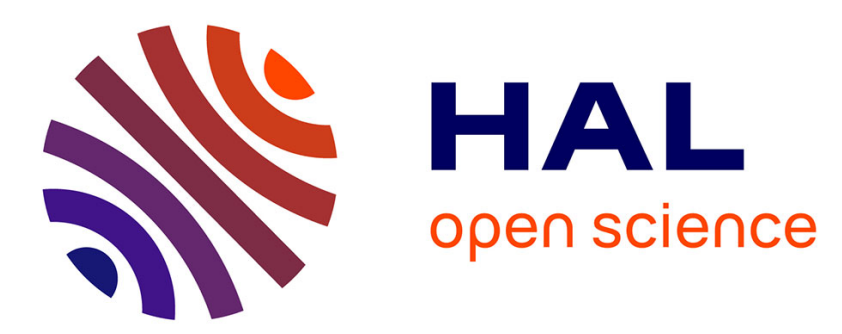

\title{
La culture de l'information au prisme des sciences de l'information et de la communication
}

\author{
Vincent Liquète
}

\section{To cite this version:}

Vincent Liquète. La culture de l'information au prisme des sciences de l'information et de la communication. Études de communication - Langages, information, médiations, 2018, 2018 (50), pp.109-128. 10.4000/edc.7607 . hal-03218615

\section{HAL Id: hal-03218615 https://hal.science/hal-03218615}

Submitted on 5 May 2021

HAL is a multi-disciplinary open access archive for the deposit and dissemination of scientific research documents, whether they are published or not. The documents may come from teaching and research institutions in France or abroad, or from public or private research centers.
L'archive ouverte pluridisciplinaire HAL, est destinée au dépôt et à la diffusion de documents scientifiques de niveau recherche, publiés ou non, émanant des établissements d'enseignement et de recherche français ou étrangers, des laboratoires publics ou privés. 


\section{La culture de l'information au prisme des sciences de l'information et de la communication}

Information Culture Through the Prism of Information and Communication Sciences

\section{Vincent Liquète}

\section{OpenEdition}

\section{Journals}

Édition électronique

URL : http://journals.openedition.org/edc/7607

DOI : 10.4000/edc.7607

ISSN : 2101-0366

\section{Éditeur}

Université de Lille

\section{Édition imprimée}

Date de publication : 1 juin 2018

Pagination : 109-128

ISBN : 978-2-917562-19-2

ISSN : $1270-6841$

\section{Référence électronique}

Vincent Liquète, «La culture de l'information au prisme des sciences de l'information et de la communication », Études de communication [En ligne], 50 | 2018, mis en ligne le 01 juin 2020, consulté le 10 décembre 2020. URL : http://journals.openedition.org/edc/7607 ; DOI : https://doi.org/10.4000/ edc. 7607 
La culture de l'information au prisme des sciences de l'information et de la communication

Information Culture Through the Prism of Information and Communication Sciences 
Depuis le début des années 2000, le champ français de la recherche en sciences de l'information et de la communication a intégré les problématiques de délimitation et de compréhension de la culture de I'information. En s'appuyant sur les archives de la revue "Études de communication ", nous présenterons quelques éléments théoriques clefs pour définir la culture de l'information. Seront ensuite exposés quelques résultats dominants permettant d'appréhender la culture de l'information en situation. Pour terminer, quelles nouvelles tendances se dessinent dans l'actualité de la recherche?

Mots-clés : culture de l'information, pratique informationnelle, usage, littératie informationnelle, représentation sociale.
By the early 2000s, the French research field of Information and Communication sciences had begun to address the fundamental issues surrounding information culture. Based on analysis of the archives of the journal Etudes de communication, we present some key theoretical elements which allow us to define information culture. We then examine the contribution of studies which consider information culture in practice, through a situational approach. Finally, we discuss some of the trends in current research on information culture.

Keywords: information culture, information practices, use, information literacy, social representation. 


\section{Introduction}

Depuis une quinzaine d'années, et plus activement encore depuis 2008 , la thématique de la culture de l'information a mobilisé de nombreux chercheurs, notamment français et francophones, issus des sciences de l'information et de la communication, ou de sciences connexes, comme les sciences de l'éducation, la psychologie cognitive ou l'anthropologie de la culture. À l'occasion de la sortie du numéro anniversaire 50 de la revue Études de Communication, nous nous sommes fixé deux objectifs au cours de cette contribution : premièrement, remettre en perspective et en questionnement la notion même de culture de l'information; deuxièmement, mettre en lumière l'apport de la revue Études de Communication et de divers contributeurs à ces questions de cultures de l'information autour d'objets et de questionnements qui nourrissent la délimitation du sujet et aide à mettre en lumière des tendances sociales fortes en matière d'usages d'information. Des premières recherches menées en France et à l'étranger à la structuration progressive du champ, on notera que depuis quelques années, chercheurs, équipes, laboratoires, revues scientifiques mettent en avant et retiennent la culture de l'information comme un des pans de leur projet scientifique. Cet intérêt partagé par les communautés scientifiques méritait donc de se retourner sur quelques années de recherche à l'occasion de ce numéro.

\section{1. \\ Quelques ancrages théoriques et épistémologiques pour appréhender et comprendre la culture de l'information}

\subsection{L'approche par les pratiques informationnelles}

La délimitation conceptuelle par l'apport théorique est centrale pour aborder les notions scientifiques, dont celle de culture de l'information. Fabien Granjon, en 2002, rappelle que " la vertu théorique des concepts ne réside pas dans le système de termes qu'ils stabilisent mais dans le travail de description et de mesure qu'ils imposent ». Parmi les concepts de référence, celui de " pratique informationnelle » a lourdement pesé sur les premiers travaux autour de la culture de l'information qui émergeront ultérieurement au courant anglo-saxon baptisé "INSU » pour "Information Needs Seeking Use ». Longtemps centrées sur le seul aspect technique des dispositifs, les analyses se sont ensuite progressivement intéressées à la manière dont ils étaient effectivement utilisés voire réappropriés. Comme le soulignent dans le numéro 35 d'Études de Communication, Madjid Ihadjadene et Stéphane Chaudiron, les études ont considéré « l'usager dans ses différentes dimensions, cognitive, psychologique, affective voire communicationnelle. Ainsi, les interactions 'homme-machine', tout comme les interactions entre individus cherchant de l'information vont 
être progressivement considérées pour devenir des thématiques centrales de compréhension des pratiques de recherches d'information en contexte et dans l'activité. Plus récemment, l'émergence de ce que A. Halavais nomme la 'search engine society' (Halavais, 2009) a conduit un troisième courant de recherches à intégrer dans son analyse le rôle de l'environnement socioéconomique dans l'accès et la maîtrise de l'information, notamment les dimensions linguistiques et culturelles, la question des inégalités face à l'information " : inégalités, fractures, etc... sont régulièrement revenues en lecture au cours de ces quinze dernières années. Dès lors, nous glissons progressivement de la seule approche interactions " hommes-systèmes ", vers une logique compréhensive des interrelations humaines autour de l'information, des documents, des dispositifs et des environnements techniques dédiés. Comme l'indiquent Cécile Gardiès, Isabelle Fabre et Viviane Couzinet (2010), en sciences de l'information et de la communication, notamment en citant Emmanuel Souchier, Yves Jeanneret et Joëlle Le Marec (2003), il s'agit de « prendre en compte l'épaisseur sociale de la pratique en construction " pour intégrer la question des contextes et saisir la complexité de ce qui est étudié. Par pratique, M. Ihadjadene rappelle qu'il s'agit de « désigner les études qui sont centrées sur l'humain (individuel ou collectif) et qui analyse ses modalités d'action (ou d'inaction), ses représentations, ses attitudes $»^{1}$.

La pierre angulaire des pratiques informationnelles en est la recherche d'information. Comme évoqué par Stéphane Chaudiron et Madjid Ihadjadene dans le numéro 35, dès 2003, Pamela McKenzie a élaboré un modèle des pratiques informationnelles visant à rendre compte de la recherche d'information "dans la vie de tous les jours », dans une analyse de la quotidienneté. Elle indique cependant les limites de sa propre étude basée sur des seuls entretiens, à savoir sans observation au cœur des situations. Pamela McKenzie toutefois ouvre une brèche en termes d'approches anthropologiques. Nous passons ainsi progressivement des habitudes de lecture aux habitudes de recherche d'information et d'évaluation de l'information avec de plus en plus en filigrane la question de la prise de décision individuelle et collective. En complément à la recherche d'information, l'évaluation de l'information constitue également un champ de recherche actif venant compléter les travaux sur la recherche d'information. En effet, l'évaluation de l'information est un temps spécifique où se confrontent recherche de données ou de documents face au capital de connaissances et/ou aux contraintes (de temps, d'argent, etc...) de l'usager. Les travaux d'Alexandre Serres (2012 notamment) en ce sens sont significatifs et ont marqué le courant français de la culture de l'information.

1 Pour S. Chaudiron et M. Ihadjadene (2010), il faut « réserver le terme d'USAGE pour désigner les travaux portant sur les dispositifs et leurs interactions avec les usagers et de réserver celui de PRATIQUE pour caractériser les approches centrées sur le comportement composite à l'œuvre dans les différentes sphères, informationnelles, culturelles, journalistiques, etc. ». 
Progressivement, les travaux sur les pratiques informationnelles prennent appui sur la démarche holistique : ainsi, les chercheurs tendent à ne plus s'intéresser « à l'utilisateur comme individu isolé face à un dispositif mais comme un usager situé dans son contexte social, culturel et linguistique " qu'il convient de décrypter. S. Chaudiron et $\mathrm{M}$. Ihadjadene soulignent dans ce numéro 35 que « plusieurs auteurs se sont attachés à intégrer les variables sociales dans leur modélisation des pratiques ».

Le volet communicationnel est lui aussi déterminant dans l'appréhension de la culture de l'information par le biais des pratiques informationnelles. Par essence, analyser la culture de l'information de manière complexe, au sens d'Edgar Morin, revient à penser la communication au sens de Meyriat (1983), à savoir que l'information est " une connaissance communiquée ou communicable, en d'autres termes le contenu cognitif d'une communication réalisée ou possible ".

En conclusion, soulignons que les concepts d'usage et de pratique informationnelle sont travaillés de longue date en particulier en sociologie, en anthropologie, en sciences de l'éducation ou en information-communication. De nombreux travaux ont contribué à repérer puis isoler des étapes clefs (recherche, évaluation, expertise, réécriture, appropriation...) et à les préciser en les inscrivant « dans la complexité de la circulation, de l'appropriation de l'information et de l'avancée des technologies numériques », nous disent C. Gardiès, I. Fabre et V. Couzinet. Les travaux sur les pratiques d'information tentent d'articuler la question de la socialisation de l'information entre les pairs ainsi que le partage des savoirs, tout en considérant les situations et les types de transfert opérés dans les situations d'affiliation entre les pairs, entre générations, etc. (Liquète, Soumagnac, 2017). Ainsi, s'intéresser aux pratiques contextualisées de l'information revient à présent à considérer la multi-dimensionnalité des pratiques informationnelles en situation. Ces analyses sont toutefois forcément réductrices de nombre de comportements, d'attitudes, de stratégies de recherche et donc simplifient une réalité sociale, cognitive et actionnelle bien plus complexe.

\subsection{Le courant du sense-making}

Ce courant nord-américain, notamment mis en lumière par Dominique Maurel dans le numéro 35 d'Études de communication de 2010, expose un ensemble d'intérêts pour appréhender et analyser les activités informationnelles. II présente le premier avantage de réfléchir à un niveau individuel, le sense-making individuel et un niveau collectif, le sense-making organisationnel. Dès lors, c'est par l'articulation de ces deux niveaux que l'on peut atteindre les niveaux de complexité des pratiques informationnelles en contexte, consistant à la fois à acquérir des habiletés et du sens par sa propre activité, tout en se confondant et en s'intégrant à des collectifs humains, qu'ils soient scolaires, professionnels, y compris dans la sphère personnelle voire intime. Le second avantage est qu'en essayant d'observer les groupes ou les organisations, l'approche 
tente de révéler comment se construit la réalité en mobilisant les processus de communication et d'interprétation des messages ou d'information (Maurel, 2010). En effet, comment se construisent, sont partagés et diffusés les savoirs au sein de collectifs humains ? Nous touchons là, nous semble-t-il, la question centrale de la construction mutuelle au sens culturel du terme. E. Weick (1995) identifie et liste ainsi 7 caractéristiques du sense-making, dont la dimension sociale s'inscrivant dans les valeurs et les interprétations partagées par un groupe d'individus qui tentent de construire une même réalité. De son côté avec l'approche individuelle du sense-making, chez Brenda Dervin, est posée la question de ce qui fait sens pour un individu et au-delà, ce qui continue de le motiver à poursuivre sa recherche d'information et à l'approprier.

Pour ce courant théorique, tout processus de construction de sens partagé passe forcément par le biais de recherche et d'appropriation de l'information, tout en s'appuyant sur les dimensions situationnelles de l'activité, et donc de la "relation aux autres ». Cependant, la discontinuité de la réalité, les cartes cognitives en jeu entre personnes, les décalages entre connaissances et activités sont autant d'obstacles à la construction en commun des savoirs et de partage. Le courant du sense-making est, nous semble-t-il, indispensable à considérer afin de résister aux tentatives de globalisation et donc d'idéalisation des processus d'appropriation des savoirs à partir des pratiques informationnelles, qui serait forcément commune, partagée et faisant sens pour tous. Autant elle constitue un socle de pratiques et de représentations pouvant être partagé entre les individus, autant les modes d'appropriation et d'intégration restent difficiles à comprendre car multiples et éclatés. Comme le souligne Dominique Maurel (2010), « la démarche individuelle pour appréhender un phénomène ne peut faire abstraction de l'influence de la collectivité ». Or, à ce stade des travaux sur les pratiques d'information, il nous semble que le modèle " centré individu " est prégnant au détriment de la considération de l'influence des contextes et des organisations dans lesquels elles se construisent.

\subsection{L'approche anthropologique des savoirs}

Ce courant porté par les anthropologues et plus largement des chercheurs en sciences humaines et sociales, nous semble fondamental pour analyser et comprendre les usages en jeu dans des contextes de recherche d'information principalement. II présente également l'avantage de nous mettre en garde contre des approches parfois mécanistes et globalisantes de pratiques sociales émergentes, complexes, parfois même paradoxales. Le numéro 42 de 2014 coordonné par Yolande Maury, Susan Kovacs et Régina Marteleto présenta l'avantage de délimiter un nouveau périmètre pour appréhender les usages et pratiques en information. Ce numéro 42 s'inscrit dans la lignée des travaux d'Annette Béguin (2009) et d'A. Béguin et S. Kovacs (2011) orienté sur l'anthropologie des savoirs documentaires. Comme le soulignent Yolande Maury et Susan Kovacs dans leur article "Étudier la part de l'humain dans les savoirs ", le " partage des valeurs et des savoirs, loin d'être un processus de 
reproduction, de propagation ou de transmission des contenus, implique une transformation, une reconfiguration des sens, des appropriations diverses et multiples » et des contournements, rajouterons-nous. Le courant de l'anthropologie des savoirs souligne l'importance de percevoir les relations entre les humains, avec des enjeux de pouvoir, de lutte ou d'influences qui sont proprement liés à la circulation des connaissances (Béguin-Verbrugge, 2009). En effet, les dimensions de pouvoir sont tout autant à considérer entre individus partageant une même culture en matière d'information, qu'entre cultures, ou pays, ou zones d'influence. Cette approche milite ainsi pour une posture de recherche qui dépasse les seules analyses théoriques, épistémologiques ou d'analyses des objets pour étudier l'homme dans sa totalité, comme socle constitutif d'une culture autour des savoirs académiques et/ou émergents. Cette approche par la " totalité complexe » repousse et critique les entrées de l'époque sur l'activité informationnelle, qui scindaient ou démultipliaient les strates de l'activité informationnelle des individus. Nous évoquerons en guise d'illustrations, les travaux cherchant à distinguer pratiques formelles/ informelles d'information, l'être social/l'être intime, la sphère scolaire/la sphère sociale ou familiale, etc. S'intéresser dès lors à la culture de l'information revient à questionner les relations humaines à l'information plus que de chercher à constituer un inventaire exhaustif des dispositifs et des moyens d'accès à l'information, supposés développer et intégrer des usages et des usagers. Comme le soulignent Y. Maury et S. Kovacs, prédomine dans cette approche, "le mode de la relation bien plus que l'inventaire".

Une autre dimension de l'anthropologie des savoirs est de dissocier objectivité/subjectivité. En effet, autant les fournisseurs, les producteurs de ressources et de dispositifs revendiquent une objectivation des pratiques sociales, autant les analyses des usages démontrent la multiplicité des subjectivités des usagers qui sur-interprètent, contournent, détournent les dispositifs et les offres pour tenter de les maitriser et de les faire leur. La construction des savoirs et des compétences informationnels s'étudie dès lors à partir de l'organisation interne des groupes tout en essayant de respecter les règles de normalisation des savoirs.

Un autre apport de cette approche est de permettre aux chercheurs de se focaliser sur les objets et les dispositifs informationnels en tant que marqueurs et outils de la pensée et de la circulation des savoirs. L'analyse de la structuration, de l'organisation et de l'indexation des ressources ne suffit plus, encore faut-il chercher à identifier et caractériser les systèmes d'intention des fournisseurs et les modes d'accompagnement voire de médiation qui lui sont liés. Dès lors, tenter d'analyser les offres équivaut pour le chercheur en sciences humaines et sociales à repérer les subjectivités en jeu par le biais des idéologies, des hiérarchies, des imaginaires, comme les travaux de définition de François Laplantine nous y invitent dès 2001. En effet, s'intéresser à l'activité informationnelle en contexte, revient à considérer, les agencements, les espaces, les pratiques (informationnelles) sociales tout en convoquant les systèmes d'ima- 
ginaire et de représentations en jeu. Claude Lévi-Strauss en 1958 indiquait que "l'anthropologue avait besoin de l'expérience du terrain »; le chercheur qui questionne la "culture de l'information » ne peut se mettre en rupture avec le terrain et les acteurs en situation d'activités et/ou de résolution de tâches. Les années 2000 à ce jour, ont ainsi vu fleurir des analyses de contextes aussi variés que le monde de l'entreprise, des décideurs, de secteurs innovants émergents, de l'espace scolaire ou universitaire..., Céline Paganelli (2016) par exemple, montre que le contexte apparaît comme une construction complexe résultant des chercheurs qui visent à comprendre des phénomènes, à partir d'une pluralité d'acception.

Enfin, l'approche anthropologique a permis de remobiliser et d'instiller, nous semble-t-il, la question des méthodologies en jeu. Car outre des postures d'observation voire d'immersion, la question de la démarche compréhensive envers les situations et environnements qui composeraient une culture de I'information revient à faire des choix de méthodes en cherchant à mobiliser progressivement des objets jusqu'alors peu considérés en sciences de l'information et de la communication. Nous citerons des journées autour du numérique avec des chercheurs en immersion ${ }^{2}$ créant des évènements avec des publics afin de tester leurs hypothèses et d'appréhender les pratiques spontanées ${ }^{3}$, des observations d'individus dans de nouveaux espaces documentaires comme les learning centers ${ }^{4}$, ou l'utilisation de la photographie ${ }^{5}$ comme moyen de recueillir des traces produites à chaud par les acteurs eux-mêmes et de les faire rétrospectivement revenir sur celles-ci lors de temps d'analyse compréhensive avec les sujets.

1.4. Une dualité de dénomination montrant une multiplicité de conceptions de la question

Ce qui peut frapper l'esprit, voire étonner tout chercheur ou professionnel qui s'intéresse à la "culture de l'information », est de constater une pluralité de nomination qui, malgré les apparences premières, cache dans la réalité une diversité de situations. Dès 2001, David Bawden tissait un lien entre culture de l'information et « culture de la culture » (cultural literacy), croisant la connaissance

2 Cf. le rapport de la tâche 2 de l'ANR Translit (2013-2016), en ligne : https:// archivesic.ccsd.cnrs.fr/CULTURES_INFORMATION/sic_01552095v1

3 Par exemple, une journée d'idéation autour du risque numérique, Bordeaux, le 12 octobre 2017 : en ligne : http://pi.espe-aquitaine.fr/journee-ideation-autour-de-la-formation-au-numerique/

4 Cf. (R)évolutions dans les bibliothèques? Les Learning Centres, un modèle de bibliothèque à interroger / Yolande Maury et al. en ligne : http://hal.univ-lille3. $\mathrm{fr} / \mathrm{hal}-01399739$

5 Cf. le travail de thèse de Valentine Mazurier intitulé «Les nouveaux territoires de la documentation scolaire : représentations et pratiques informationnelles des collégiens " en cours de réalisation, où des collégiens photographient des situations ou des objets caractérisant selon eux l'espace documentaire qu'ils vivent dans leur quotidien d'élèves. 
des normes et des valeurs d'une culture en relation avec les textes classiques qui la définissent, et la "culture de l'informatique " combinant maîtrise de l'ordinateur et accès aux divers réseaux plus ou moins disponibles et ouverts. Le vocable " culture de l'information " se voulait dès lors être générique et à l'articulation entre littératies traditionnelles et littératies numériques plus instables et en perpétuelle modification. Ainsi, la culture de l'information associerait donc des savoirs académiques relativement stables et partagés de tous, donc institutionnalisés, avec des savoirs plus dynamiques liés à l'essor de l'informatique, du numérique et des industries de la connaissance, davantage centrés sur des habiletés et des capacités d'action. Elle articule également les usages et la capacité à utiliser des technologies d'information et/ou de communication, dans la lignée des analyses de Claude Baltz, dès 1998. De son côté, Christine Bruce (1997), dans une perspective "information literacy » qualifie la culture de l'information comme un outillage intellectuel et cognitif créant des prédispositions à se former et s'auto-former tout au long de la vie permettant à la fois de renforcer un équilibre et renforcement des savoirs à l'échelle individuelle, tout en assurant l'essor économique et innovant d'un territoire à un niveau collectif. De ces influences nord-américaines, les premiers chercheurs français s'intéressant à la culture de l'information sont dans le paysage des sciences de l'information et de la communication, plutôt issus du champ de l'information-documentation, et plutôt enclin à se centrer sur les organisations médiatrices des savoirs que sont les bibliothèques, les centres de documentation, les CDI, etc. C'est notamment le cas de la recherche nationale ERTé « Culture informationnelle et curriculum documentaire » coordonnée par Annette Béguin qui débute dès 2006 et organise un colloque international à Lille en 2008. Ensuite, en 2009, Olivier Le Deuff, dans son travail de thèse doctorale, présente une première forme de formalisation scientifique de cette notion, en délimitant la culture de l'information à l'articulation des domaines technologies, biblio-documentaire, agrégeant des habiletés issues des secteurs d'activité observée. II rend également hommage à la définition antérieure de Michel Menou (2008) définissant la culture de l'information comme " un système de valeurs, d'attitudes et de comportements, de connaissances et d'aptitudes qui conduisent non seulement à un usage intelligent et approprié de l'information externe, mais surtout à contribuer à la diffusion et à la bonne utilisation de l'information tant externe qu'interne (ou produite/reconfigurée par soi-même). Donc une culture de partage et d'enrichissement collectif ». On soulignera une entrée davantage centrée sur l'humain et ses interactions aux autres, que sur les techniques et leur essor dans notre quotidienneté.

Plus récemment, émerge une seconde notion à savoir la culture informationnelle : deux volets la caractérise et la dissocie de la culture de l'information telle que présentée. D'une part, la culture informationnelle conduirait à une attitude responsable et citoyenne permettant aux individus une plus grande et meilleure intégration sociale, ou scolaire, ou culturelle, ou professionnelle. Dès lors, l'intention est moins la recherche de l'opérationnalité des pratiques 
que la dimension analytique, critique voire de résistance aux contextes d'offres de l'information. Sur ce point, elle se démarque de la culture de l'information, qui selon le propos de Brigitte Juanals, en 2013, écrivait qu'il était important d'aller « au-delà de la seule vision d'une maîtrise technologique et méthodologique de l'accès à l'information pour tendre vers une culture de l'information nécessitant à la fois une culture générale, une réflexion et une éthique ". D'autre part, la culture informationnelle prend particulièrement en considération, les contextes d'exercice de l'activité et des usagers plus ou moins formels. À la différence de la culture de l'information fortement anthropo-centrée, la culture informationnelle suggère une analyse des écosystèmes d'information et les interrelations et appropriations de l'individu avec eux. Ainsi, l'approche par la culture informationnelle s'articule avec l'essor de l'éducation à l'information où il devient également nécessaire de différencier le courant de la maîtrise de I'information avec des études sur les phénomènes culturelles avec l'information (Maury, 2013).

\section{2. \\ Cultures de l'information et cultures des organisations}

\subsection{Les organisations scolaires et universitaires}

Force est de constater que depuis une quinzaine d'années, l'essentiel des publications sur la culture de l'information s'intéresse prioritairement aux sphères scolaires et universitaires. Pour le secteur scolaire, ce sont plus exactement les élèves des établissements du second degré, collège et lycée d'enseignement général voire professionnel, qui sont considérés, notamment à travers le prisme de dispositifs d'enseignement singuliers, comme les travaux croisés à une époque, plus récemment les Travaux Personnels Encadrés (TPE), les Itinéraires de Découvertes (IDD), les "éducations à... ", ou l'Éducation aux médias et à I'Information (EMI) dernièrement. Étonnamment, les travaux sur les formes émergentes de culture de l'information chez les plus jeunes, en classes maternelles (avec les pré-lectures et pré-écritures) ou primaires, ne sont pas encore réellement convoqués, à l'exception de quelques travaux sur les documents en tant que considération des matérialités documentaires en tant qu'entrée dans le monde de la culture de l'information, selon le principe de trivialisation au sens d'Yves Jeanneret (Béguin, Kovacs, 2011). Certainement également, parce que les premières recherches doctorales menées puis soutenues autour de ce périmètre le sont par des professeurs-documentalistes exerçant exclusivement dans le second degré ou dans l'enseignement supérieur ${ }^{6}$. Ainsi, 
une grande part des travaux autour des cultures de l'information ont eu pour caractéristique de se focaliser sur des pratiques d'information centrées avant tout sur le document (la pédagogie documentaire au sens d'Annette Béguin), puis sur les formes et les obstacles dans l'appropriation des environnements numériques documentaires de travail, ou l'entrée des notions et des concepts informationnels dans les apprentissages par le biais des approches didactiques de l'information.

Ces divers travaux font cependant à I'heure actuelle ressortir un ensemble de constats qui semblent relativement partagés par les chercheurs du domaine. Nous en retiendrons cinq :

- une place de choix et pré-déterminante du sentiment d'expertise chez les apprenants (Cordier, 2015). Les représentations des uns et des autres tendent à valoriser le numérique comme cadre de référence, aussi bien chez les jeunes que chez les adultes dont le discours institutionnel est dominé par l'injonction technologique, sans prise en compte de la réalité des obstacles cognitifs, ni des modèles et des méthodes d'apprentissage véritablement en jeu. Sans pour autant que les enseignants plébiscitent le numérique, puisqu'ils semblent encore privilégier le papier et les documents traditionnels ;

- les démarches de culture de l'information au cours des activités pédagogiques plus ou moins encadrées, révèlent une hybridation d'activités oscillant entre le " papier ", les documents traditionnels et les offres numériques disponibles depuis le système d'information de l'établissement scolaire ou via les réseaux (Internet, réseaux sociaux numériques...). On voit alors poindre des objets documentaires, marqueurs d'ancrage de pratiques pour les cultures de l'information : les systèmes arborescents, les cartographies et leurs variantes, les systèmes d'indexation en langage contrôlé ou naturel, etc ;

- I'organisation autour de dispositifs centrés « pédagogie par projet » faciliterait les interactions entre apprenants et entre apprenants et professeurs, de telle manière qu'ils favoriseraient un cadre opératoire pour le développement et l'apprentissage de connaissances et de compétences en matière d'information (lecture, analyse, recherche, sélection, évaluation, réécriture, production documentaire...). Ces projets s'appuient également sur un ensemble d'espaces et de modes d'organisation plus souples et variés que la classe, facilitant ainsi les échanges, les co-constructions et la production de contenus voire de ressources. Cette dimension-là 
questionne voire remet en question les modes d'organisation actuelle de la classe ;

- favoriser et soutenir la culture de l'information, revient aussi pour les enseignants à lutter contre les idées reçues et disséminées socialement de générations spontanées constituant un unanimisme médiatique autour de concepts slogans, dénoncé par exemple, par Pascal Lardellier (2017) : génération $Y$, digital natives, App génération, etc. sont autant d'appellations de surface qui ne renvoient à aucune pratique sociale partagée de tous, tant celles-ci sont variées et reposent sur des systèmes d'intention variables ;

- enfin, les projets numériques centrés sur la recherche et la production d'information, structurent une véritable organisation collective du travail, négociée entre les pairs, en s'appuyant sur les compétences des uns et des autres pour optimiser la démarche. Ce mode d'organisation collectif des savoirs, interroge dès lors, l'équilibre des acquisitions entre les apprenants, nécessitant pour l'enseignant de s'assurer pédagogiquement que chacun rééquilibre ses déficits de compétences plutôt que de les amplifier.

\subsection{Les organisations entrepreneuriales}

À l'inverse, les travaux s'inscrivant sur l'analyse compréhensive des cultures de l'information en contexte d'entreprise, au niveau micro ou macro-social, sont encore rares en France et lorsqu'ils prennent en considération ce type de terrains, ne se centrent pas sur les cultures de l'information mais plutôt les pratiques informationnelles, ou d'écriture collaborative autour de collectifs de salariés. Ce qui nous incite à dire que la faible prise en compte de la part des chercheurs des cultures de l'information dans le contexte entrepreneurial mériterait d'être analysé voire ajusté. Les scientifiques s'intéressant aux entreprises, quel que soit le domaine d'activités ou leur taille, cherchent avec tout à capter les pratiques d'écriture et de partage (Bessières, 2012), ou, centrent leurs études sur les modes d'organisation de la communication interne voire de la prise de décision. Ainsi, le courant de l'anthropologie des savoirs a occupé une place de choix, par le biais notamment de l'ethnographie des pratiques et l'analyse des énoncés notamment autour des écrits professionnels. Nous pensons aux travaux de Béatrice Fraenkel, de Sylvie Grosjean, etc. Cette dernière, dans sa contribution au numéro 42 d'Études de communication, restitue une analyse de données recueillies par l'observation directe et en temps réel des activités qui se déploie au sein d'une compagnie d'arpenteur-géomètre. S. Grosjean au cours de ce travail montre comment un professionnel organise son terrain afin de "faire corps " avec lui et de pouvoir anticiper ses actions futures. Objets, outils, éléments d'information sont ainsi organisés en vue d'organiser au mieux l'activité professionnelle à venir. Les descriptions méticuleuses des tâches et des traces nous montrent un entrelacement d'éléments langagiers, sensibles, de données (issues de GPS) et de prises de note variées. Ces approches en 
contexte révèlent le tuilage et l'agrégation de formes expérientielles avec les données, les écrits et des objets matériels d'information (feuilles, appareils numériques etc.).

Ainsi, les approches autour des communautés des savoirs et de la théorie de l'activité permettent de capter et d'analyser la culture de l'information dans les contextes professionnels, notamment en entreprise. L'approche des " communautés de savoirs » à travers Lave (1988), Wenger (2002), ou plus récemment Cox (2005), caractérise pour eux une communauté dans cette approche pragmatique où s'effectue un engagement mutuel des membres du groupe, c'est-à-dire qu'il y a un ensemble d'accords tacites entre eux pour être solidaires et échanger un ensemble d'informations susceptibles de revêtir une utilité future par anticipation et projection. Pour ce courant, les communautés de savoir ne sont pas toujours dans des logiques informatives de concurrence mais plutôt de partage et de mutualisation quand il y a conscience d'un intérêt à terme commun et de retombées à l'échelle individuelle. Étienne Wenger enrichit cette approche, en évoquant l'existence de répertoires cachés facilitateurs du dialogue entre les pairs. Ce dernier suppose dans son approche, que toute communauté s'appuie sur des activités avec les documents, mobilisant un ensemble de ressources et de gisements, constituant ainsi des répertoires cachés où s'échangent des descriptions de «bonnes pratiques » et un ensemble d'activités de co-écriture. La difficulté pour le chercheur est de pouvoir atteindre ces répertoires cachés qui n'ont pas vocation à être rendus disponibles aux personnes extérieures à l'organisation ou au projet professionnel.

Plus personnellement, nos travaux dans divers secteurs professionnels, en immersion, comme les agriculteurs indépendants, les acteurs de l'éco-construction notamment, nous ont permis d'appréhender deux dimensions obstacles à la culture de l'information dans l'activité professionnelle. D'une part, plus qu'une difficulté à rechercher et trouver l'information, car les professionnels ont une connaissance approfondie de leur domaine d'activité, la difficulté pour eux est de faire face à des modes de diffusion du document et de l'information qu'ils ne peuvent ou pas acquérir pour des raisons économiques ou gérer au sein d'une pluralité de discours, qui polluent et diluent leur registre d'intention en tant que concepteur et constructeur. D'autre part, l'autre difficulté est qu'ils ne peuvent concevoir la connaissance sans considérer les divers enchevêtrements de réseaux ; nous avons affaire à des réseaux extrêmement complexes à décortiquer combinant la fourniture d'informations tout autant que des modes de communication et d'échanges permettant d'asseoir la culture professionnelle de l'information de leur domaine d'activités. 


\section{3. \\ Quelques perspectives de recherches sur les cultures de l'information}

\subsection{Continuer d'interroger les pratiques informationnelles en situation}

Force est de constater qu'une part des études menées autour des pratiques informationnelles qui se rapprocheraient d'une considération des cultures de I'information, en tant que composantes de celle-ci, se sont souvent affichées voire ont revendiqué des dichotomies que la réalité des pratiques en situation ont critiqué. Parmi les dichotomies repérées nous en énoncerons trois: d'une part, la séparation pratiques sociales vs pratiques professionnelles (ou scolaires) ; d'autre part, la dichotomie pratiques personnelles vs pratiques collectives ; enfin, pratiques formelles vs pratiques informelles (ou moins formelles). Depuis le début des années 2010, les approches autour des translittératies ${ }^{7}$ ont présenté le mérite de remettre en question ces séparations artificielles d'usages complexes, croisant les espaces, les temporalités et les dispositifs entre eux, tout en considérant les capitaux culturels et sociaux (Bourdeloie, 2012).

Ainsi, les observations menées dans les espaces scolaires ou associatifs ont montré que le cadre de référence des publics observés n'est ni l'espace ni la technique en soi mais plutôt le lien social qui se créait aux cours des temps de travail, de co-écriture et de résolution de tâches. De plus, le lien supposé entre les trois cultures de l'information, celle de l'informatique, celle du documentaire et celle du médiatique, n'est pas évident. La plupart des individus observés mettent plutôt l'accent sur une des trois littératies et se divisent à plusieurs la résolution des taches de travail et de création. Ce qui semble primer dans la relation, est la rencontre des autres et de leurs pratiques, plutôt que le rendu final, l'évaluation ou l'amélioration des activités menées. Dans les contextes associatifs à forte prescription, les pratiques créatives et multimodales sont censurées par les acteurs eux-mêmes; le poids des médiateurs numériques jouent prioritairement ce rôle de régulateur qui affaiblit la part de créativité et de production. Ainsi, la culture de l'information semble plutôt s'articuler entre information-documentation et pratiques des médias de masse, qu'entre compétences numériques/informatiques et documentation ou médias. Enfin, le tuilage entre les différentes littératies supposé par de nombreuses modélisations autour de la translittératie reste à ce jour encore extrêmement fragile; la figure ci-dessous donne un exemple de modélisation, issue de I'ANR Translit : 


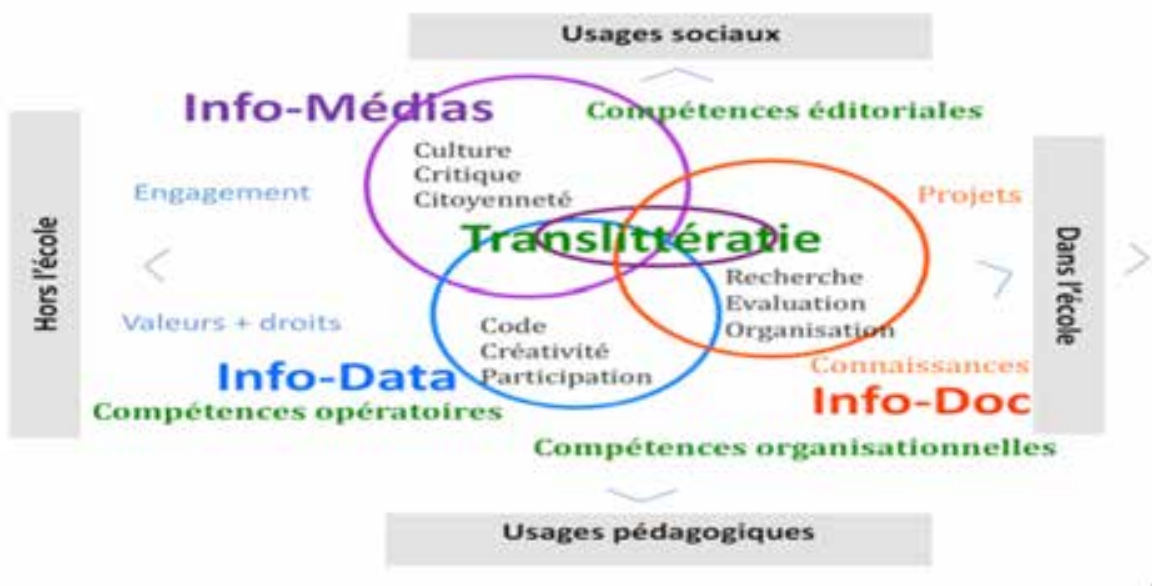

Figure 1

Toutefois, la force de l'approche par croisement des cultures mass-médiatiques, numériques et documentaires, est de parvenir à distinguer trois registres actifs de compétences :

- les compétences éditoriales qui se centrent principalement sur la culture des sources et la compréhension des conditions de production et de traitement des informations de masse (Kervella, Loicq, 2015) ;

- les compétences organisationnelles qui consistent à organiser la tâche, en mobilisant un ensemble de techniques et de dispositifs permettant de traiter l'information principalement recherchée et recueillie : indexation, synthèse, condensation et traduction prioritairement ;

- les compétences opératoires qui visent à savoir éditorialiser ses propres résultats de recherche et de réécriture, en s'appuyant sur le codage, la programmation, la connaissance des algorithmes, etc.

Ces dimensions pourraient constituer un des socles d'appréhension de la culture de l'information.

\subsection{Vers une culture des données}

Alors même que, comme nous avons tenté de le montrer, l'approche des cultures de l'information est loin d'être délimitée, nous voyons poindre à présent de nouvelles formes de d'opérations contemporaines de la culture de l'information en action, autour de la recherche, de la manipulation et de la conservation de données, qui reposent à la fois sur des compétences de classement (sur la base des folksonomies et des pratiques populaires d'indexation) et d'analyse générale des types de données. On notera progressivement que le vocable "donnée » a épousé celui de l'information. Cette émergence de la 
culture de la donnée est particulièrement portée par un double mouvement, celui de l'institution scolaire qui prescrit depuis peu, la question des données dans les programmes d'enseignement du lycée, par exemple, en sciences économiques et sociales, ou en géographie. L'incitation sociale devient alors une injonction ministérielle. D'autre part, car un champ de la donnée a cristallisé les attentes des enseignants, celui de la gestion des données personnelles (Georges, 2010) et des traces : l'enjeu est alors d'identifier et de décrypter "la manière dont on conduit la conduite des hommes", comme l'indique Bernhard Rieder dans le numéro 35 d'Études de communication (2010). Force est de constater que l'entrée de la donnée dans les organisations, notamment scolaires, va venir enrichir mais complexifier la question de la culture de l'information (Lehmans, 2017).

\section{Conclusion}

Comme nous avons tenté de le démontrer au cours de ces quelques pages, le chantier de la recherche autour des cultures de l'information est loin d'être stabilisé ou achevé. Outre que ce domaine articule des niveaux politiques, sociaux, cognitifs complexes, la réalité des terrains d'observation et les démarches méthodologiques retenues donnent à voir des tendances sociales encore multiples, variées et forcément fragiles voire contradictoires. Les enjeux en termes d'usages, de pratiques et d'acquisition sont au cœur de la thématique des cultures de l'information. Nous voyons également poindre, tant en matière d'écrits scientifiques, que de nouvelles recherches financées et/ou doctorales des perspectives venant enrichir et compléter des réalités d'activités informationnelles encore peu connues ou interrogées. Nous avons dès lors bien à faire à une notion vivante, en cours de délimitation théorique et pratique. La revue Études de Communication a ainsi tout au long de la période considérée, depuis 2010, accompagné de nombreux chercheurs et laboratoires sur la question de la genèse et de l'essor des travaux sur la culture de l'information. Consulter et sélectionner des articles dans ses archives, revient à traverser cette question de la relation, de la recherche et de l'appropriation de savoirs à partir d'informations (voire de données). La revue y a apporté une réelle plus-value scientifique dans ce champ émergent de la recherche en sciences humaines et sociales. 
Bibliographie

Baltz C. (1998). « Une culture pour la société de l'information? Position théorique, définition, enjeux ». In Documentaliste-Sciences de l'information, vol. $35, n^{\circ} 2$, p. 75-82.

Bawden D. (2001). « Information and Digital Literacies: A Review of Concepts ". In Journal of Documentation, vol. $57, n^{\circ} 2$, p. 218-259.

Béguin A., Kovacs S. (dir.) (2011). Le cahier et l'écran, culture informationnelle et premiers apprentissages documentaires, Paris, Hermès-Lavoisier.

Béguin-Verbrugge A. (2009). "Information, Communication et Anthropologie des savoirs ". In RECIIS - Revista Eletrônica de Comunicação, Informação e Inovação em Saúde, vol. 3, n³, p. 35-41. Disponible sur https://www.arca.fiocruz. br/bitstream/icict/17493/2/5.pdf (page consultée le 23 janvier 2018).

Bessières D. (2012). " Sociologie de l'appropriation des TICE : peut-on parler d'une culture informationnelle partagée ou de genèse d'usage ? ". In Études de communication, $\mathrm{n}^{\circ} 38$, p. 37-52.

Bourdeloie H. (2012).

«L'appropriation des dispositifs d'écriture numérique : translittératie et capitaux culturel et social ». In Études de communication, $\mathrm{n}^{\circ} 38$, p. 23-36.
Bruce C. S. (1997). Seven

faces of information literacy, Adélaïde, Auslib Press.

Chaudiron S., Ihadjadene M. (2010). « De la recherche de I'information aux pratiques informationnelles ». In Études de communication, $\mathrm{n}^{\circ} 35, \mathrm{p} .13-30$.

Cordier A. (2015). Grandir connectés. Les adolescents et la recherche d'information, Caen, C\&F éditions.

Couzinet V. (2008a). Vers « une société du savoir » : approche ethno-informationnelle de la " culture de l'information ». In The scientific annals of "Alexandru loan Cuza ", vol. 1, $\mathrm{n}^{\circ}$ 1. Disponible sur http:// anale.fssp.uaic.ro/index.php/stiintealecomunicarii/article/view/280 (page consultée le 2 octobre 2017)

Couzinet V. (2008b). « De l'usager à l'initié : vers une culture informationnelle partagée ». In Éducation à l'information et éducation aux sciences : quelles formes scolaires ? Actes des rencontres Educagro, p. 169-189.

Cox A. (2005). «What are communities of practice? A comparative view of four seminal works ". In Journal of Information Science, vol. 31, $n^{\circ}$ 6, p. 527-540.

Delamotte E., Liquète V., FrauMeigs D. (2014). « La translittératie, ou la convergence des cultures de l'information : supports, contextes et modalités $»$. In Spirale, $n^{\circ}$ 53, p. 145-156. 
Gardiès C., Fabre I., Couzinet V. (2010). « Re-questionner les pratiques informationnelles ». In Études de communication, $n^{\circ} 35$, p. 121-132.

Georges F. (2010). «Pratiques informationnelles et identités numériques ». In Études de communication, $\mathrm{n}^{\circ} 35$, p. 105-120.

Granjon F. (2002). «Les sciences de I'information et de la communication toujours à la recherche de leur(s) spécificité(s) ». In Les recherche en information et communication et leurs perspectives : histoire, objets, pouvoir, méthodes. Actes $d u 13^{e}$ congrès national des sciences de l'information et de la communication, p. 409-415.

Grosjean S. (2014). «Étudier la dimension sensible des savoirs produits en contexte de travail ». In Études de communication, $\mathrm{n}^{\circ} 42$, p. 47-62.

Halavais A. (2009). Search Engine Society, Cambridge, Polity press.

Juanals B. (2003). La culture de I'information : du livre au numérique, Paris, Lavoisier, Hermès sciences.

Kervella A., Loicq M. (2015). « Les pratiques télévisuelles des jeunes à l'ère du numérique : entre mutations et permanences ". In Études de communication, $\mathrm{n}^{\circ} 44$, p. 79-96.

Laplantine F. (2001). L'anthropologie, Paris, Ed. Payot et Rivages.

Lardellier P. (2017), “' ' $Y$ ' et digital natives, faux concepts et vrais slogans. Une lecture critique de deux 'ressources sûres' de la doxa numérique ». In Hermès, n 78, p. 151-158.

Lave J. (1988). Cognition in Practice: Mind, mathematics, and culture in everyday life, Cambridge, UK, Cambridge University Press.

Le Crosnier H. (coord.) (2013). Culturenum : jeunesse, culture \& éducation dans la vague numérique, Caen, C\&F éd.

Le Deuff O. (2009), La culture de l'information en reformation, Sciences de l'Homme et Société, Thèse à I'Université Rennes 2, sous la direction $\mathrm{d}^{\prime} Y$. Chevalier. Disponible sur https://tel.archives-ouvertes.fr/file/index/docid/421928/ filename/theseLeDeuff.pdf (page consultée le 1er octobre 2017).

Le Deuff O. (2012). « Littératies informationnelles, médiatiques et numériques : de la concurrence à la convergence? ». In Études de communication, $\mathrm{n}^{\circ} 38$, p. 131-147.

Lehmans A. (2017). « Données ouvertes et redéfinition de la culture de l'information dans les organisations ". In Communication et organisation, $n^{\circ} 51$, p. 15-26.

Liquète V., Soumagnac K. (dir.) (2017). Les affiliations par et avec le numérique, Paris, Hermann.

MacKenzie P. (2003). “A model of information practices in account of everyday life information seeking". In Journal of Documentation, vol. 59, $n^{\circ} 1$, p. 19-40. 
Maurel D. (2010). « Sense-making : un modèle de construction de la réalité et d'appréhension de l'information par les individus et les groupes ». In Études de communication, $n^{\circ} 35$, p. 31-46.

Maury Y. (2013). « De la culture de l'information à la culture informationnelle : au-delà du 'penser, classer, catégoriser' ». In Frisch M. (dir.), Nouveaux espaces et dispositifs en question, nouveaux horizons en formation et en recherche : objets de recherche et pratiques "en écloserie », Paris, L'Harmattan, p. 125-148.

Maury Y., Kovacs S. (2014). «Étudier la part de l'humain dans les savoirs : les Sciences de l'information et de la communication au défi de l'anthropologie des savoirs ». In Études de communication, $n^{\circ} 42$, p. 15-28.

Maury Y., Serres A. (2010). "Les cultures informationnelles : définitions, approches, enjeux ». In Chapron F., Delamotte E. (dir.), L'Education à la culture informationnelle, Actes du Colloque international de l'ERTé, Françoise Chapron et Eric Delamotte (dir.), Villeurbanne, ENSSIB , p. 26-39.

Menou M. (2008). « Culture de I'information ». In Cacaly S., Le Coadic Y.-F., Pomart P.-D., Sutter E. (dir.), Dictionnaire de l'information, $3^{e}$ éd., Paris, Armand Colin, p. 65-67.

Meyriat J. (1983). « De la science de l'information aux métiers de l'information ». In Schéma et schématisation, $n^{\circ} 19$, p. 65-74.
Paganelli C. (2016). « Réflexions sur la pertinence de la notion de contexte dans les études relatives aux activités informationnelles ». In Études de communication, $n^{\circ} 46$, p. 165-188.

Kerr Pinheiro M. M., Thiesen I., Couzinet V. (2008). « Choc informationnel et culture de l'information : quelle formation à l'information? ». In Couzinet V., Chaudiron S. (dir.), Sciences de la société, $n^{\circ} 75$, p. 141-158.

Pinto M., Sales D. (2007). "Alfabetización informacional para una sociedad intercultural: algunas iniciativas desde las bibliotecas pùblicas". In Anales de documentación, n 10, p. 317-333.

Rieder B. (2010). «Pratiques informationnelles et analyse des traces numériques : de la représentation à l'intervention ». In Études de communication, $n^{\circ} 35$, p. 91-104.

Serres A. (2012). Dans le labyrinthe. Evaluer l'information sur Internet, Caen, C\&F éditions.

Souchier E., Jeanneret Y. et Le Marec J. (2003). Lire, écrire, réécrire : objets, signes et pratiques des médias informatisés, Paris, BPI.

Weick K. E. (1995). Sensemaking in organization, Thousand oaks, CA, Sage Publications.

Wenger E., Mac Dermott R., Snyder W. M. (2002). Cultivating Communities of Practice: a Guide to Managing Knowledge, Boston, Harvard Business School Press. 


\section{Références Études de} communication

Études de communication, Dossier Pratiques informationnelles : questions de modèles et de méthodes, $\mathrm{n}^{\circ} 35,2010$.

Études de communication, Dossier L'éducation à l'information, aux TIC et aux médias : le temps de la convergence?, $\mathrm{n}^{\circ} 38,2012$.

Études de communication, Dossier Anthropologie des savoirs, $n^{\circ} 42,2014$.

Études de communication, Dossier Pratiques télévisuelles à l'ère du numérique, $\mathrm{n}^{\circ}$ 44, 2015. 